\title{
Diagnóstico de Enfermagem em Unidade de Terapia Intensiva Adulto
}

\author{
Andreza Pinto Aires da Costa*, Ana Elza Oliveira de Mendonça, M.Sc.**
}

*Enfermeira, Pós-graduada em Enfermagem em UTI pela Faculdade União Americana, **Enfermeira, Professora do Departamento de Enfermagem da UFRN, Doutoranda em Ciências da Saúde CCS/UFRN, Enfermeira intensivista do HUOL/ $U F R N$

\section{Resumo}

Atualmente a Sistematizaçáo da Assistência de Enfermagem (SAE), em especial o Diagnóstico de Enfermagem, vem sendo implementado como forma de proporcionar uma melhor assistência aos pacientes e valorização do profissional enfermeiro. Porém esta implementaçáo tem encontrado dificuldades em todo âmbito assistencial. O objetivo do presente estudo foi pesquisar na literatura, trabalhos que versem sobre a aplicação do diagnóstico de enfermagem em UTI. A presente revisão foi realizada nas bases eletrônicas de dados indexadas a Biblioteca Virtual em Saúde (BVS), Literatura Latino-Americana e do Caribe em Ciências da Saúde (Lilacs) e Base de Dados de Enfermagem (BDENF), utilizando para a localização dos artigos o cruzamento dos seguintes descritores: "Diagnóstico de Enfermagem" e "Unidade de Terapia Intensiva". Ao total foram selecionados cinco artigos, que atenderam aos critérios de inclusão estabelecidos: idioma, intervalo de tempo e formato. Identificou-se que existe pouca produção literária sobre o assunto e dificuldade de aceitação entre os profissionais, ficando um estímulo a publicaçóes sobre o tema e incentivo a capacitação dos profissionais sobre o assunto.

Palavras-chave: diagnóstico de enfermagem, sistematização da assistência de enfermagem, unidades de terapia intensiva.

\section{Abstract}

\section{Nursing diagnosis in Adult Intensive Care Unit}

Currently the Nursing Care Systematization (NCS), in particular the Nursing Diagnosis, is developed, as a way to provide better patient care and enhancement of the professional nurse. But this implementation has encountered difficulties throughout health care. The objective of this literature review was the application of nursing diagnosis in the ICU. This review was performed in electronic databases indexed in the Virtual Health Library (VHL), the Latin American and Caribbean Health Sciences (Lilacs) and Database of Nursing (BDENF), using to locate articles the intersection of the following descriptors: "Nursing Diagnosis" and "Intensive Care Unit". Altogether five articles were selected that met the established inclusion criteria: language, time frame and format. It was identified that there is little literature on the subject and difficulty of acceptance among professionals, and that publications on the subject should be encourage as well as professional training on the subject.

Key-words: nursing diagnosis, nursing care system, intensive care units. 


\section{Resumen}

\section{Diagnóstico de Enfermería en Unidad de Terapia Intensiva Adulto}

En la actualidad la Sistematización de la Asistencia de Enfermería (SAE), en particular el Diagnóstico de Enfermería, ha sido implementado para proporcionar mejor atención al paciente y perspectivas del profesional de enfermería. Sin embargo, esta implementación ha encontrado dificultades en todo el ámbito asistencial. El objetivo de este estudio fue buscar en la literatura estudios relacionados con la aplicación de diagnósticos de enfermería en la UCI. Esta revisión se realizó en bases de datos electrónicas indexadas en la Biblioteca Virtual en Salud (BVS), América Latina y el Caribe en Ciencias de la Salud (Lilacs) y Base de Datos de Enfermería (BDENF), utilizando los siguientes descriptores: "Diagnóstico de Enfermería" y "Unidad de Cuidados Intensivos" para localizar los artículos. En total, se seleccionaron cinco artículos que cumplían los criterios de inclusión establecidos: el lenguaje, el marco temporal y el formato. Se ha concluido que existe poca literatura sobre el tema y dificultad de aceptación entre los profesionales, y que se debe estimular publicaciones sobre el tema, así como fomentar la formación de profesionales en el asunto.

Palabras-clave: diagnóstico de enfermería, sistematización de asistencia de enfermería, unidades de cuidados intensivos.

\section{Introdução}

A Sistematização da Assistência de Enfermagem (SAE) como forma de proporcionar uma melhor assistência aos pacientes, bem como valorizar o profissional enfermeiro, tem tentado ser implementado em todo o âmbito assistencial de saúde no Brasil. Porém, tem-se encontrado muitas dificuldades para alcançar esse objetivo.

A SAE é composta pela documentação das etapas do processo de enfermagem, a fase do histórico, do diagnóstico de enfermagem, do planejamento e a avaliação de enfermagem [1]. Esta divisão tem cunho apenas didático, uma vez que na prática assistencial a SAE é um processo com etapas inter-relacionadas e dinâmico [2].

O Diagnóstico de Enfermagem, uma parte da SAE e segundo a North American Nursing Association (NANDA), consiste no julgamento clínico sobre as respostas do indivíduo, da família ou da comunidade, a problemas de saúde/processos vitais reais ou potenciais. $\mathrm{O}$ diagnóstico de enfermagem proporciona a base para seleção de intervençóes de enfermagem, para atingir resultados pelos quais a enfermeira é responsável [3].

A enfermagem tem se aproximado dos sistemas de classificaçáo com o intuito de se afastar do referencial que lhe guiou nas últimas décadas: o das técnicas e do tratamento curativo/interventivo e descontextualizado. Busca-se um referencial centrado no cuidado, específico, embasado em novos saberes, valores, conhecimentos e contextualizados [4].
Aponta-se também que o uso de uma linguagem padronizada é uma das prioridades da profissão, seja no ensino, na pesquisa ou na assistência, uma vez que torna visível e reconhecido o saber e o fazer da enfermagem pelas demais áreas do conhecimento [5].

Nesse contexto, faz-se necessário o desenvolvimento de uma gestão do cuidado com vistas ao alcance de melhores resultados, que oportunize o desenvolvimento de habilidades para implantação do processo de enfermagem. Dessa forma, é preciso estimular e sensibilizar a equipe de enfermagem assistencial acerca da sua aplicabilidade e decorrente obtenção de resultados, tanto para o paciente como para a instituiçáo [6].

Frente a essa realidade e considerando que a gestão do cuidado cada vez mais está voltada para a obtenção de melhores resultados na prática clínica, estratégias têm sido desenvolvidas para a minimização e controle desses fatores intervenientes. Já há evidências de que a quantidade e a qualidade da documentação têm apresentado expressiva melhora nos prontuários [7].

O conhecimento do perfil dos pacientes internados torna-se importante para direcionar as açóes de cuidado, a busca pelo conhecimento e a melhoria ou reformulação na implementaçáo do processo de enfermagem. Nesse sentido, a análise dos registros de enfermagem em prontuários pode contribuir para a identificaçáo de necessidades e resultados obtidos em decorrência de processos de educação permanente em saúde [8]. 
O conjunto de diagnósticos de enfermagem, que um paciente (ou uma determinada clientela) apresenta, evidencia tanto a complexidade de seu quadro clínico e, consequentemente, o tipo de intervençóes requeridas para resolução dos mesmos como, também, o respectivo conjunto de atividades necessárias, expressas nas prescriçôes de enfermagem [9].

Em um ambiente de terapia intensiva, as necessidades de cuidado de enfermagem são mais complexas, pois os pacientes exigem avaliaçóes críticas e rápidas, planos de cuidados abrangentes e de serviços bem coordenados com outros profissionais da saúde [10].

Acredita-se que o conhecimento acerca da aplicabilidade dos diagnósticos de enfermagem em unidades de terapia intensiva entre os profissionais ainda é precário, cabendo as instituiçóes empregadoras incentivar e colaborar para programas de educação permanente.

Desta forma, o objetivo do estudo foi identificar na literatura artigos que versem sobre a aplicação ou implementação do diagnóstico de enfermagem em unidades de terapia intensiva adulto.

\section{Material e métodos}

Trata-se de um estudo de revisão de literatura do tipo narrativa. A revisão narrativa sintetiza e re- sume, em termos narrativos, um corpo e literatura de pesquisa [11].

As bases de dados utilizadas foram a Literatura Latino-Americana e do Caribe em Ciências da Saúde (Lilacs) e Base de Dados de Enfermagem (BDENF), cruzando os descritores da Biblioteca Virtual de Saúde (BVS), "Diagnóstico de Enfermagem" e "UTI". A coleta de dados se deu no mês de outubro de 2011, abrangendo publicaçóes referentes ao período de 2005 a 2011.

Têm-se como critérios de inclusão: Artigos originais, completos e publicados em revista de enfermagem, no idioma de português e que abordassem a temática do estudo. Os critérios de exclusão foram: teses de mestrado, artigos incompletos, artigos de não acesso gratuito, não condizentes com o tema $\mathrm{e}$ fora do intervalo de tempo.

Foram identificados 05 artigos, na base Lilacs, porém destes, 02 artigos são comuns a base BDENF. Os quais foram lidos na íntegra, sumarizados, resumidos em quadros para posterior discussão.

Os artigos que fizeram parte da amostra foram apresentados em forma de quadro com as seguintes informações: título, autores, periódico, ano de publicação e objetivo.

\section{Resultados e discussão}

Os artigos selecionados para a pesquisa estão descritos no Quadro 1, a seguir:

Quadro 1 - Descrição dos artigos científicos trabalhados.

\begin{tabular}{|l|l|l|l|l|}
\hline \multicolumn{1}{|c|}{ Título } & \multicolumn{1}{|c|}{ Autores } & Periódico & $\begin{array}{c}\text { Ano de } \\
\text { publicação }\end{array}$ & \multicolumn{1}{c|}{ Objetivos } \\
\hline $\begin{array}{l}\text { Implantação do Diag- } \\
\text { nóstico de Enfermagem } \\
\text { em Unidade de Terapia } \\
\text { Intensiva: uma análise } \\
\text { periódica [6] }\end{array}$ & $\begin{array}{l}\text { Paganin A, } \\
\text { Klafke T, } \\
\text { Lazzarotto A, } \\
\text { Fachinelli TS, } \\
\text { Chaves IC, Souza EN. }\end{array}$ & $\begin{array}{l}\text { Rev Gaúch } \\
\text { Enferm }\end{array}$ & 2010 & $\begin{array}{l}\text { Identificar os principais diagnósticos } \\
\text { de enfermagem na UTI e compará- } \\
\text {-los entre os pacientes clínicos e } \\
\text { cirúrgicos. }\end{array}$ \\
$\begin{array}{l}\text { Sistematização da Assis- } \\
\text { tência de Enfermagem } \\
\text { em Unidade de Terapia } \\
\text { Intensiva [12] }\end{array}$ & $\begin{array}{l}\text { Truppel TC, Meier MJ, } \\
\text { Calixto RC, Peruzzo } \\
\text { AS, Crozeta K. }\end{array}$ & Rev Bras Enferm & 2009 & $\begin{array}{l}\text { Reestruturar a SAE em uma UTI; elen- } \\
\text { car os diagnósticos e as prescrições de } \\
\text { enfermagem; validar as etapas da SAE } \\
\text { e subsidiar a estruturação de um proto- } \\
\text { colo para operacionalização da SAE. }\end{array}$ \\
\hline
\end{tabular}




\begin{tabular}{|l|l|l|l|l|}
\hline \multicolumn{1}{|c|}{ Título } & \multicolumn{1}{c|}{ Autores } & Periódico & \multicolumn{1}{c|}{$\begin{array}{c}\text { Ano de } \\
\text { publicação }\end{array}$} & \multicolumn{1}{c|}{ Objetivos } \\
\hline $\begin{array}{l}\text { Diagnóstico de ne- } \\
\text { cessidades da família } \\
\text { de clientes adultos na } \\
\text { unidade de terapia } \\
\text { intensiva: revisão de } \\
\text { literatura [14] }\end{array}$ & $\begin{array}{l}\text { Ribeiro JA, Santos } \\
\text { MSS. }\end{array}$ & $\begin{array}{l}\text { Rev Cogitare } \\
\text { Enferm }\end{array}$ & 2008 & $\begin{array}{l}\text { Descrever as necessidades apre- } \\
\text { sentadas pelos familiares à luz dos } \\
\text { diagnósticos de enfermagem. }\end{array}$ \\
\hline $\begin{array}{l}\text { Relações entre a coleta } \\
\text { de dados, diagnósticos e } \\
\text { prescrições de enferma- } \\
\text { gem a pacientes adultos } \\
\text { de uma unidade de } \\
\text { terapia intensiva [15] }\end{array}$ & $\begin{array}{l}\text { Carvalho EC, Martins } \\
\text { FTM, Dalri MCB, } \\
\text { AM, Bachion MM, } \\
\text { Rossi LA. }\end{array}$ & $\begin{array}{l}\text { Rev Latinoam } \\
\text { Enfermagem }\end{array}$ & 2008 & $\begin{array}{l}\text { Analisar os registros de enfermagem } \\
\text { tendo como foco o perfil de diagnós- } \\
\text { ticos de enfermagem identificados; } \\
\text { analisar a relação das prescrições de } \\
\text { enfermagem aos elementos constitu- } \\
\text { tivos dos diagnósticos identificados; } \\
\text { analisar o diagnóstico de enferma- } \\
\text { gem de maior ocorrência, em relação } \\
\text { à sua sustentação nos dados de } \\
\text { avaliação do paciente e a pertinência } \\
\text { de prescrições a ele estabelecidas. }\end{array}$ \\
\hline
\end{tabular}

Fonte: Dados da pesquisa.

Foram avaliados os 5 artigos na íntegra, em que se pode verificar que os diagnósticos de enfermagem (DE) em UTI, de modo geral, são quase os mesmos. Os DE encontrados em comum nos artigos em maiores números estáo listados no Quadro 2.

Quadro 2 - Diagnóstico de Enfermagem comum nos artigos.

\begin{tabular}{|l|c|}
\hline \multicolumn{1}{|c|}{ Diagnósticos de enfermagem } & $\begin{array}{c}\text { Comum nos } \\
\text { artigos }\end{array}$ \\
\hline Integridade tissular prejudicada & $6,13,15$ \\
\hline Perfusão tissular cerebral ineficaz & 6,12 \\
\hline Ventilação espontânea prejudicada & 12,13 \\
\hline Padrão respiratório ineficaz & 12,13 \\
\hline Risco para aspiração & 12,15 \\
\hline Risco para infecção & 13,15 \\
\hline Mobilidade física prejudicada & 13,15 \\
\hline
\end{tabular}

Fonte: Dados da pesquisa.

O diagnóstico de integridade tissular prejudicada está relacionado à presença de ferida operatória, após cirurgia; tempo prolongado no leito sem a mudança de decúbito, o banho, administração de medicamentos (via subcutânea). A maior dificuldade encontrada no leito de UTI é a do paciente não ser capaz de realizar atividades por si próprias, portanto, requerendo intervençóes que proporcionem as atividades da vida diária do indivíduo e promovam o seu conforto $[6,13,15]$.

A perfusão tissular cerebral ineficaz refere-se a uma situação que se caracteriza por mudanças nas rea- çóes pupilares, no estado mental, nas respostas motoras e de comportamento. Todas essas manifestaçooes clínicas indicam redução do fluxo sanguíneo, interferindo na oxigenação e nutrição do parênquima cerebral [12]. Como também pode ser identificado em pacientes submetidos à cirurgia cardíaca e, consequentemente, recuperando em uma unidade de intensivismo, nesses pacientes ocorrem grandes alteraçóes no padrão respiratório e função ventilatória, afetando vários sistemas, inclusive a perfusão cerebral [6].

Os diagnósticos de ventilação espontânea prejudicada e o padrão respiratório ineficaz são definidos como o funcionamento anormal ou incompleto do movimento do ar para dentro e para fora dos pulmóes com certo padrão e ritmo respiratório, profundidade de inspiração e força de expiração. Observa-se que estes diagnósticos se relacionam à mecânica da ventilação e não aos processos de troca gasosa e transporte de oxigênio/dióxido de carbono. Os riscos para estes diagnósticos são o posicionamento inadequado do paciente, posicionamento errado de sondas e drenos gastrintestinal, acúmulo de secreçóes no trato respiratório, ventilação mecânica ineficaz $[12,13]$.

Risco para aspiraçáo ocorre em pacientes com risco de inalação de substâncias exógenas ou gástricas para as vias aéreas inferiores. Como pode ocorrer no posicionamento inadequado da sonda gástrica, pacientes submetidos a cirurgias que náo se encontram em jejum [12,15]. 
O diagnóstico de enfermagem de risco para infecção está agrupado na Taxonomia II da NANDA no domínio 11, que trata de Segurança/Proteção, significando "estar livre de perigo, lesóes físicas ou danos no sistema imunológico, prescrição contra perdas e proteção da segurança e seguridade" e na classe 1 infecções " que são as respostas do hospedeiro após a invasão patogênica”. Os fatores de risco para este diagnóstico são procedimentos invasivos, destruição de tecidos, doença crônica, agente farmacêutico, exposição ambiental a patógeno, defesa primária inadequada, defesa secundária inadequada, incontinência e retenção urinária [13,15].

Mobilidade física prejudicada é o risco quando o paciente náo pode deambular ou mudar de posição devido a algum motivo, que pode ser encontrado em pacientes submetidos principalmente a cirurgias de grande porte, pacientes sob ventilação mecânica, posicionamento inadequado no leito ou cadeira de rodas, paciente com dor, úlceras de pressão $[13,15]$.

Ribeiro e Santos [14] aborda os diagnósticos de enfermagem para as necessidades das famílias de pacientes internados em UTI. As análises construídas foram: A interdependência da comunicação e relacionamento terapêutico para efetivação do cuidado na UTI; vulnerabilidade do cliente e da família e seus desdobramentos na saúde; cumprimento do papel da família esperado pelas enfermeiras no cuidado ao cliente; e a filosofia institucional e o distanciamento do profissional de saúde da família na UTI.

A partir da análise do estudo, foi possível verificar que os Diagnósticos de Enfermagem ainda não são amplamente utilizados, enfrentando dificuldades para sua implantação nos ambientes de assistência hospitalar.

Com a comparação dos artigos, pode-se concluir que alguns diagnósticos são comuns no ambiente de Unidade de Terapia Intensiva, já que os riscos aos quais os pacientes estáo expostos de um modo geral são quase os mesmos.

\section{Conclusão}

Foi perceptível que há uma deficiência na literatura com relaçáo a trabalhos publicados sobre Diagnósticos de Enfermagem, ficando uma lacuna que se espera ser preenchida com informaçóes e análises positivas do processo de utilização de implementação da Sistematização da Assistência de Enfermagem.

\section{Referências}

1. Andrade JS, Vieira MJ. Prática assistencial de enfermagem: problemas, perspectivas e necessidade de sistematização. Rev Bras Enferm 2005;58(3):261-5.

2. Conselho Federal de Enfermagem (BR). Resoluçáo n ${ }^{\circ}$ 272/2002, Dispóe sobre a Sistematização da Assistência de Enfermagem - SAE - nas Instituiçôes de Saúde Brasileiras. In: Truppel TC, Meier MJ, Calixto RC, Peruzzo SA, Crozeta K, eds. Sistematização da assistência de enfermagem em unidade de terapia intensiva. Rev Bras Enferm 2009;62(2):221-7.

3. North American Nursing Diagnosis Association (NANDA). Diagnósticos de enfermagem: definiçóes e classificaçóes - 2003-2004. Porto Alegre: Artmed; 2005. p.283.

4. Antunes MJM, Chianca TCM. As classificações de enfermagem na saúde coletiva: o projeto CIPESC. Rev Bras Enferm 2002;55(6):644-51.

5. Crossetti MGO, Dias V. Utilização da classificação na prática e no ensino de enfermagem: experiência brasileira. Rev Bras Enferm 2002;55(6):720-4.

6. Paganin A, Menegat P, Klafke T, Lazzarotto A, Fachinelli TS, Chaves IC, Souza EM. Implantaçáo do diagnóstico de enfermagem em unidade de terapia intensiva: uma análise periódica. Rev Gaúcha Enferm 2010;31(2):307-13.

7. Daly JM, Buckwalter K, Maas M. Written and computadorized care plans. J Gerontol Nurs 2002;28(9):1423.

8. Muller-Staub M. Evaluation of the implementation of nursing diagnoses, interventions, and outcomes. Int J Nurs Terminol Classif 2009;20(1):9-15.

9. Helberg JL. Patients' status at home care discharge. Image J Nurs Sch 1993;25(2):93-9.

10. Vargas RS, França FCV. Processo de enfermagem aplicado a um portador de cirrose hepática utilizando as terminologias padronizadas NANDA, NIC E NOC. Rev Bras Enferm 2007;60(3):348-52.

11. Polit DF, Beck CT, Hungler BP. Fundamentos da pesquisa em enfermagem: métodos, avaliação e utilização. 5a ed. Porto Alegre: Artmed; 2004. 487p.

12. Truppel TC, Meier MJ, Calixto RC, Peruzzo SA, Crozeta K. Sistematização da assistência de enfermagem em unidade de terapia intensiva. Rev Bras Enferm 2009;62(2):221-7.

13. Lucena AF, Gutiérrez MGR, Echer IC, Barros ALBL. Intervenções de enfermagem utilizadas na prática clínica de uma unidade de terapia intensiva. Rev Latinoam Enferm 2010;18(5).

14. Ribeiro JA, Santos MSS. Diagnóstico de necessidades da família de clientes adultos na unidade de terapia intensiva: revisão de literatura. Cogitare Enferm 2008;13(3):437-42.

15. Carvalho EC, Martins FTM, Dalri MCB, Canini SRMS, Laus AM, Bachion MM, Rossi LA. Relaçôes entre coleta de dados, diagnósticos e prescriçôes de enfermagem a pacientes adultos de uma unidade de terapia intensiva. Rev Latinoam Enferm 2008; 16(4):700-6. 